\title{
Discriminative properties of shock-correlated reinforcement in an operant context*
}

\author{
JOHN G. CARLSON and RICHARD M. WIELKIEWICZ \\ Lniversity of Hawail. Honolulu, Hawaii 96822
}

A two-choice discrete operant procedure was devised for the study of shock-correlated reinforcement effects in rats. In the presence of one auditory stimulus, responding on one response lever was reinforced with food; with another auditory stimulus, responding on a second lever was reinforced. It was found that discrimination performance of one group, relative to appropriate control groups, was facilitated when electric shock was correlated with reinforcement on one lever and not on the other. Further, relative discrimination levels were found to be higher on the lever correlated with the shock than on the alternate lever. The significance of the results for operant within-S studies and for a mediational theory of shock-correlated reinforcement was discussed.

An extensive line of research has shown that the addition of electric shock to reinforcement may facilitate simple discrimination learning (cf. Fowler \& Wischner. 1969). The typical procedure involves exposure of one group of animals to a single-choice T-maze task in which electric shock is followed by food in one arm and not in the other arm of the maze. Relative to another group that receives no shock in either arm, correct responding of the former group is typically enhanced.

On the basis of this research it seems reasonable to suppose that effects of shock-correlated reinforcement may also be obtained within Ss. However, such a demonstration would require a somewhat more sophisticated discrimination task than has been employed in this area-for instance, a task that provides two reinforced responses, one shocked and the other unshocked. If used in conjunction with a technique which would more readily lend itself to automation than traditional T-maze procedures, a greater variety of variables related to shock-correlated reinforcement effects may be subject to study by investigators in this area.

In the present experiment, two-choice leverpressing of rats with respect to external stimuli was established, utilizing dry food reinforcement. For respective groups, a mild electric shock was delivered at the time of reinforcement for one, both, or neither "correct" response. This procedure afforded a means to assess differential effects of the shock upon the groups as well as upon responding of Ss on the two response levers

\footnotetext{
* Based on a paper presented by the first author at the annual meeting of the Midwestern Psychological Association, Chicago, 1973. Computer services were provided by the University of Hawaii Computing Center. Reprints may be obtained from John G. Carlson. Department of Psychology, University of Hawaii, Honolulu, Hawaii 96822.
}

within the group shocked on just one lever. Automatic retraction and insertion of the response levers allowed for convenient scheduling of multiple trials within sessions.

\section{METHOD \\ Subjects}

Twenty-four male albino rats, approximately 80 days old at the start of the study, were maintained at $80 \%$ of free-feeding body weight on a daily portion of Lab Chow.

\section{Apparatus}

A Lehigh Valley operant chamber was equipped with two retractable response levers spaced $85 \mathrm{~mm}$ to each side of a food trough, a pellet feeder that dispensed single $45-\mathrm{mg}$ Noyes food pellets as reinforcements, an electrifiable grid floor, houselight, and loudspeaker. Continuous white noise in the experimental room masked the sound of control and recording equipment in another room.

The animals were adapted to the chamber, magazine trained, and leverpress trained. Then. for two sessions, the two levers were presented individually and on a random schedule at varying intervals, averaging $21 \mathrm{sec}$. On each "trial," a lever would enter the chamber and the first response delivered reinforcement and caused the lever to be retracted. After 30 reinforcements on each lever, the session was terminated and the houselight extinguished. On the third session and in all remaining sessions of this phase, a scrambled electric shock $(0.7 \mathrm{~mA}$ on the scale of a BRS 901 shock generator) was presented for $0.02 \mathrm{sec}$ at the delivery of reinforcement on a random half of the trials on each lever. ${ }^{1}$ On the fourth and each succeeding session, the requirement for reinforcement was increased by one leverpress until a maximum of 10 presses was established. Then nine additional sessions at the 10-response level were given.

Following this pretraining, the animals were assigned to three groups of equal size and placed on a two-lever discrimination problem. At varying intervals averaging $21 \mathrm{sec}$, timed from the end of the preceding trial, either a tone (approximately $1,000 \mathrm{~Hz}$ ) or click (approximately $6 \mathrm{pps}$ ) was sounded. Three seconds later, both levers appeared in the chamber. Ten responses on the right lever in the presence of the tone and 10 responses on the left lever in the presence of the click stimulus delivered reinforcement, retracted the lever, and terminated the auditory stimulus for the duration of the intertrial interval. The 10 th response on the alternate "incorrect" lever in each auditory stimulus was followed by retraction of the lever and termination of the auditory stimulus without reinforcement. In addition, for half of the animals in the correlated-shock group, each reinforcement in the presence of the tone was accompanied by the electric shock. For the remaining animals in this group, each reinforcement in the presence of the click was correlated with the shock. In the random-shock group, the electric shock was presented with reinforcement on a random half of the trials on each lever. For the no-shock group, no shocks were delivered at any time. There were 30 tone and 30 click trials in each of 24 sessions.

\section{RESULTS}

Figure 1 shows means of daily percentages of correct responses across both response levers. Throughout the 


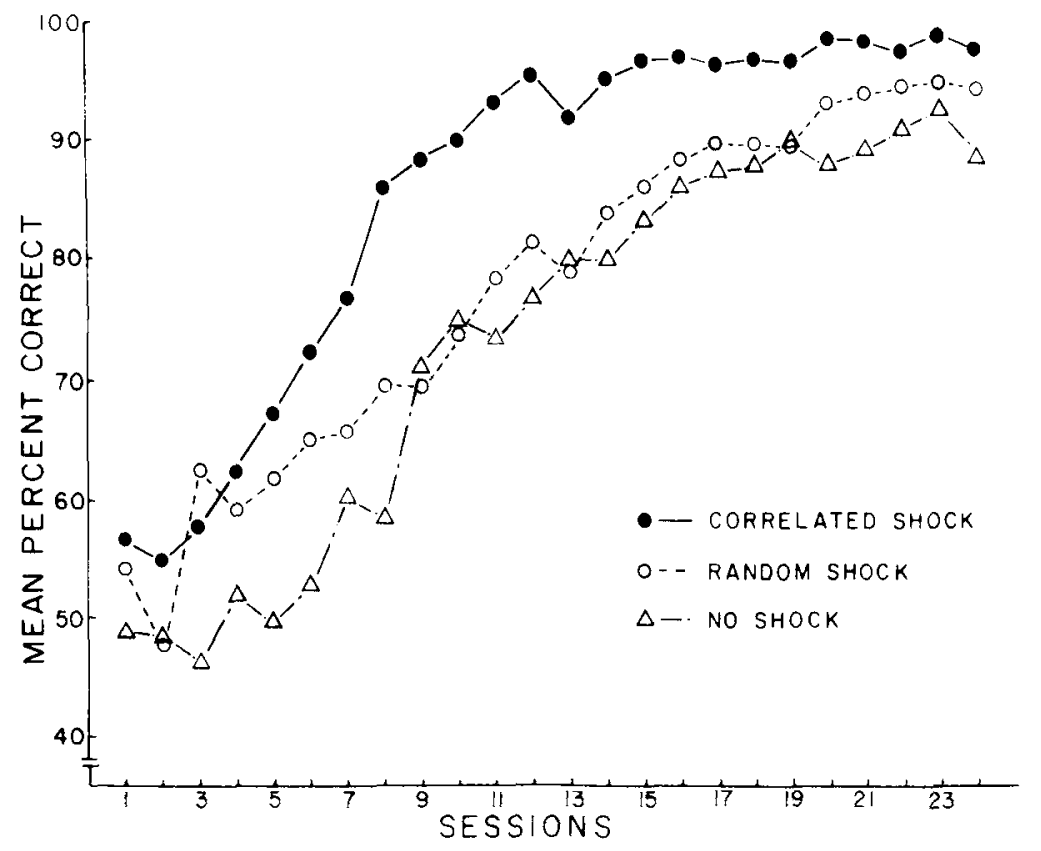

Fig. 1. Mean percentages of correct re sponding of the three groups across both response levers.

greater part of discrimination training, the group for which the shock was randomly correlated with reinforcement on both levers performed at about the same level as the no-shock group. Both of these groups gradually attained a high level of performance $(85 \%$ correct and better) within 16 sessions. By contrast, the group for which shock was correlated with just one of the two responses (and external stimuli) acquired the discrimination somewhat more rapidly, emitting more than $85 \%$ correct responses within 8 sessions. An analysis of variance on correct responses with groups and sessions as factors yielded a significant groups effect $(\mathrm{F}=$ 7.84 , df $=2 / 21, p<.005)$, a Groups by Sessions interaction $(F=1.51, \mathrm{df}=46 / 483, \mathrm{p}<.025)$, and a sessions effect $(F=75.68$, df $=23 / 483, p<.0005)$. Follow-up individual comparisons on the group means paralleled the trends apparent in the curves. The random-shock and no-shock group means did not differ from one another $(.05<p<.10)$ and each was smaller than the correlated-shock group mean $(\mathrm{p}<.025, .001$, respectively).

An examination of daily performance on each lever within the correlated-shock group revealed an initial preference for the no-shock lever in terms of a greater number of correct and incorrect responses. Therefore, discrimination levels on each lever were determined by computing daily ratios of correct responses to total responses on each lever for every animal. The means of these ratios for the group are shown in Fig. 2. Discrimination performance on the shock lever clearly exceeded that on the no-shock lever in the first 12 sessions and equaled or slightly exceeded that on the no-shock lever in 11 of the 12 remaining sessions. An analysis of variance on these data, with levers and

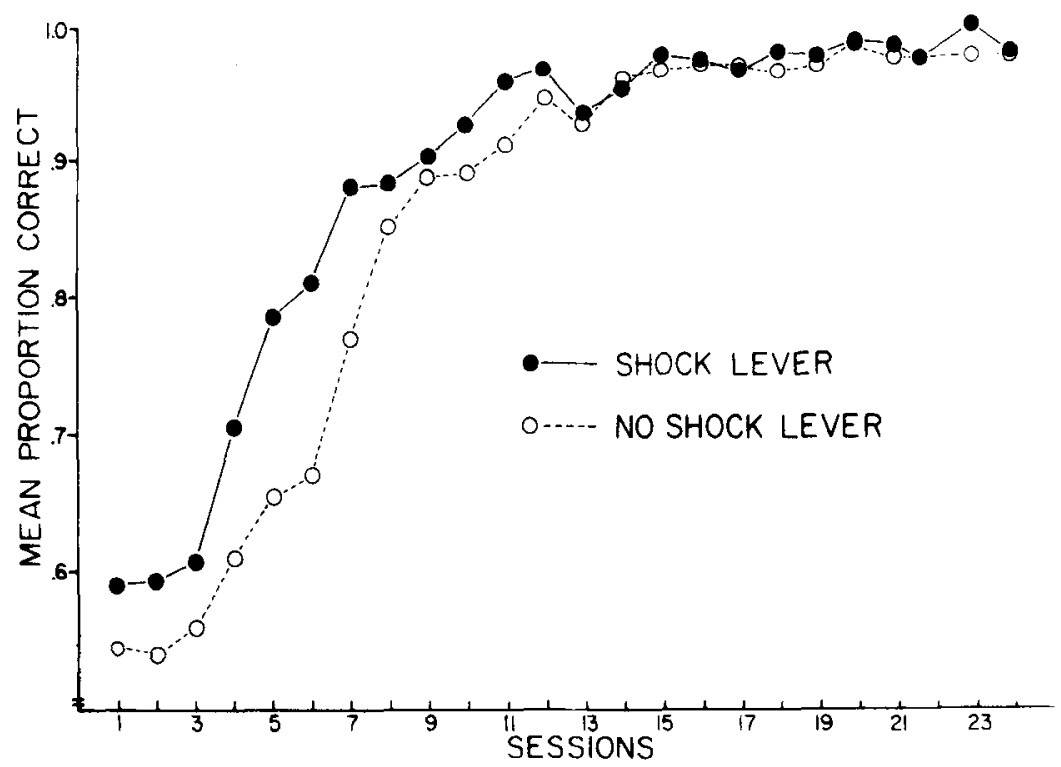

Fig. 2. Mean number of correct responses as a proportion of total responses on each response lever in the correlated-shock group. 
sessions as factors, yielded a significant lever effect $(F=$ 11.39. $\mathrm{df}=1 / 7, \mathrm{p}<.025)$, a Lever by Sessions interaction $(\mathrm{F}=1.83$, df $=23 / 161, \mathrm{p}<.025)$, and a sessions effect $(F=29.96, \mathrm{df}=23 / 16, \mathrm{p}<.0005)$.

\section{DISCUSSION}

The higher performance levels of the correlated-shock group relative to the other groups is an effect analogous to that typically obtained in traditional single-choice T-maze settings. Of even greater interest, within the correlated-shock condition relative performance levels on the shock lever were reliably higher than on the no-shock lever, despite the animals' initial preference for the no-shock lever and consequent greater number of reinforcements and nonreinforcements on this lever.

From a methodological standpoint, this research demonstrates a powerful discrete-trial operant technique for the study of shock-correlated reinforcement. The two-choice procedure lends itself to within-S analyses at the same time that the operant characteristics of the setting provide commonly cited advantages over conventional maze settings. This should give impetus to investigators who wish to extend earlier analyses of the many factors related to shock-correlated reinforcement effects.

From a theoretical standpoint, an extensive discussion of hypotheses concerning the effects of shock-correlated reinforcement is provided by Fowler and Wischner (1969). In our view, both the between-group and within-S effects of the shock are consistent with a mediational interpretation which would invoke associative rather than motivational concepts. As a result of the correlation between one of the auditory stimuli and the shock, the animals in the correlated-shock group may have come to anticipate the shock in the presence of this stimulus and not in the presence of the alternate stimulus. Then at the beginning of each trial, anticipation-related stimuli would have been available to add to the complex of stimuli impinging upon these animals. The other groups would not have had this advantage, since in either case similar anticipations would have been conditioned to both auditory cues due to the similarity of the reinforcement conditions across trials. Motivational effects of the shock were not apparent, since the group shocked on both levers (random shock) performed at approximately the same level as the no-shock group. Also contrary to expectations from a motivational view, the correlated-shock group performed at a lower level on the shock lever than on the no-shock lever in terms of absolute numbers of responses. In general, these results are comparable to those obtained when parameters of positive reinforcement are manipulated under similar conditions (e.g., Carlson \& Wielkiewicz, 1972; Trapold, 1970), a parallel which implicates a fundamental mediational mechanism in operant learning based on either positive or negative reinforcers.

\section{REFERENCES}

Carlson, J. G., \& Wielkiewicz, R. M. Delay of reinforcement in instrumental discrimination learning of rats. Journal of Comparative \& Physiological Psychology, 1972, 81, 365-370.

Fowler, H., \& Wischner, G. J. The varied functions of punishment in discrimination learning. In B. A. Campbell and R. M. Church (Eds.), Punishment and aversive behavior. New York: Appleton-Century-Crofts, 1969. Pp. 375-420.

Trapold, M. A. Are expectancies based upon different positive reinforcing events discriminably different? Learning \& Motivation, 1970, 1, 129-140.

\section{NOTE}

1. The shock intensity was established through direct observation of a random sample of the Ss. The level chosen was sufficient to evoke a clear low amplitude jump in all of the observed animals. No residual emotionality was apparent in terms of the ease with which the animals could be handled following each experimental session or the readiness with which the animals consumed food pellets following shock.

(Received for publication September 18, 1973; revision received November $21,1973$. 